\title{
Existence of Solutions of Second Order Boundary Value Problems with Integral Boundary Conditions and Singularities
}

\section{Guoju Ye and Xiuli Li}

Department of Mathematics, Hohai University, Nanjing 210046, China

Correspondence should be addressed to Guoju Ye, guojuye@163.com

Received 28 December 2009; Accepted 20 March 2010

Academic Editor: Yuming Xing

Copyright (c) 2010 G. Ye and X. Li. This is an open access article distributed under the Creative Commons Attribution License, which permits unrestricted use, distribution, and reproduction in any medium, provided the original work is properly cited.

By the notation and monotone convergence theorem of Henstock-Kurzweil integral, we investigate the existence of continuous solutions for the second order boundary value problems with integral boundary conditions in which the nonlinearities $f(t, x(t))$ are allowed to have the singularities in $t$ and are not Lebesgue integrable.

\section{Introduction}

The singular boundary value problems

$$
\begin{gathered}
-x^{\prime \prime}=f(t, x), \quad t \in(0,1), \\
\alpha x(0)-\beta x^{\prime}(0)=0, \\
\gamma x(1)+\delta x^{\prime}(1)=0,
\end{gathered}
$$

where $f(t, x)$ may be singular at $t=0$ and $t=1$, have been studied extensively; see, for example, [1-8], and the references contained therein.

In [7], Taliaferro showed that problem $(1.1)$ has a $C[0,1] \cap C^{1}(0,1)$ solution, where $\beta=0, \delta=0$, and $f(t, x)=q(t) x^{-\lambda}, \lambda>0, q \in C(0,1)$ with $q>0$ and $\int_{0}^{1} t(1-t) q(t) d t<+\infty$.

Since then, there are many improvements of this result in literatures for more general case. 
In [5] and other literatures, the authors studied (1.1) in the case where $f(t, x)=$ $q(t) g(x), g:[0, \infty) \rightarrow[0, \infty)$ is continuous, and $q \in C(0,1)$ with $\int_{0}^{1} t(1-t) q(t) d t<+\infty$ or in the case where $f(t, x):(0,1) \times R \rightarrow R$ is continuous and satisfies $|f(t, x)| \leq h(t)$ with $h \in C\left((0,1), R^{+}\right)$and $\int_{0}^{1} t(1-t) q(t) d t<+\infty$. We note that $f(t, x)$ admit a time singularity at $t=0$ and /or $t=1$ and space singularity at $x=0$.

In [4], the authors considered (1.1) when $\beta=0, \delta=0, f(t, x)=q(t) g(x), g:[0, \infty) \rightarrow$ $[0, \infty)$ is continuous, and $q \in L^{1}(0,1), q(t) \geq 0$ a.e. (in particular, $q$ is allowed to have a finite number of singularities).

In [1], Agarwal and O'Regan studied (1.1) when $\beta=0, \delta=0$, and $f(t, x)$ satisfies the following caratheodory conditions.

$\left(C_{1}\right)$ The map $x \mapsto f(t, x)$ is continuous for a.e. $t \in[0,1]$.

$\left(C_{2}\right)$ The map $t \mapsto f(t, x)$ is measurable for all $x \in R$.

$\left(C_{3}\right)$ There exists $h \in L_{\text {loc }}^{1}(0,1)$ with $\int_{0}^{1} t(1-t) h(t) d t<+\infty$ such that $|f(t, x)| \leq h(t)$ for a.e. $t \in[0,1]$ and $x \in R$.

In [8], the authors studied (1.1) with $\beta=0$ as well as $\delta=0$ and supposed that $f(t, x)=$ $f_{1}(t, x)+q(t), f_{1}(t, x):(0,1) \times R \rightarrow R$ is continuous, and $q \in L^{1}(0,1)$.

It is noticed that the case

$$
f(t, x)=f_{1}(t, x)+2 \sin \frac{1}{t}-\frac{2}{t} \cos \frac{1}{t}-\frac{1}{t^{2}} \sin \frac{1}{t}
$$

with $f_{1}(t, x):(0,1) \times R \rightarrow R$ being continuous is not included in all those papers abovementioned.

In this paper, motivated by this case, relying on theory of Henstock-Kurzweil integral, we investigate the following second order boundary value problems with integral boundary conditions

$$
\begin{gathered}
-x^{\prime \prime}=f(t, x), \quad t \in(0,1), \\
x(0)-k_{1} x^{\prime}(0)=\int_{0}^{1} h_{1}(s, x(s)) d s, \\
x(1)+k_{2} x^{\prime}(1)=\int_{0}^{1} h_{2}(s, x(s)) d s,
\end{gathered}
$$

where $k_{1}, k_{2}$ are nonnegative constants and $f(t, x), h_{i}(t, x)(i=1,2)$ are not certainly $L^{1}$ integrable.

Henstock-Kurzweil integral encompasses the Newton, Riemann and Lebesgue integrals. A particular feature of this integral is that integrals of highly oscillating function which occur in quantum theory and nonlinear analysis such as $F^{\prime}(t)$, where $F(t)=t^{2} \sin t^{-2}$ on $(0,1]$ and $F(0)=0$, can be defined.

For the literatures in which the theory of Henstock-Kurzweil integral to study differential equations is used we refer to [9-14] and so on.

This paper is organized as follows. In Section 2, we make some preliminaries in Henstock-Kurzweil integral; in Section 3, we will prove the equivalence of problem (1.3) and an integral equation as well as existence and uniqueness of solution for the linear problem 
which associate with (1.3); in Section 4, we are devoted to the existence results for the singular problem (1.3). An example will be given in Section 5.

\section{Preliminaries}

In this section we introduce the basic facts on Henstock-Kurzweil integrability, a concept that extends the classical Lebesgue integrability on the real line. All notations and properties can be found in the references (see, e.g., $[13,14]$ ).

Let $[0,1]$ be the real unit interval provided with the $\sigma$-algebra $\Sigma$ of Lebesgue measurable sets with the Lebesgue measure $\mu$.

Definition 2.1 (see $[13,14]$ ). One says that $D=\left\{\left(I_{i}, t_{i}\right)\right\}$ is a tagged partition of $[0,1]$ if $\left\{I_{i}\right\}$ is a finite family of closed subintervals $I_{i}$ of $[0,1]$ which are nonoverlapping, that is, their interiors are pairwise disjoint, and whose union is $[0,1]$, and if $t_{i} \in I_{i}$. Given a function $\delta:[0,1] \rightarrow(0, \infty)$ (called a gauge of $[0,1])$, one says that a tagged partition $D=\left\{\left(I_{i}, t_{i}\right)\right\}$ is $\delta$-fine if $I_{i} \subset\left(t_{i}-\delta\left(t_{i}\right), t_{i}-\delta\left(t_{i}\right)\right)$ for every $i$.

Definition 2.2 (see $[13,14]$ ). A function $f:[0,1] \rightarrow R$ is said to be Henstock-Kurzweil (shortly HK) integrable if there exists a real $z$ satisfying that, for every $\varepsilon>0$, there is a gauge $\delta_{\varepsilon}$ such that

$$
\left|\sum_{i=1}^{n} f\left(t_{i}\right) \mu\left(I_{i}\right)-z\right|<\varepsilon
$$

for every $\delta_{\varepsilon}$-finite partition $D=\left\{\left(I_{i}, t_{i}\right)\right\}$. One says that

$$
z=(\mathrm{HK}) \int_{0}^{1} f(t) d t
$$

is a Henstock-Kurzweil (shortly HK) integral of $f$ over $[0,1]$.

A function $F$ is absolutely continuous (or $\mathrm{AC}^{*}$ ) on $E \subset[0,1]$ if for each $\epsilon>0$ there exists $\delta>0$ such that $\sum_{i} \omega\left(F ;\left[c_{i}, d_{i}\right]\right)<\epsilon$ whenever $\left\{\left[c_{i}, d_{i}\right]\right\}$ is a finite collection of nonoverlapping intervals that have endpoints in $E$ and satisfy $\sum_{i}\left(d_{i}-c_{i}\right)<\delta$ while $\omega\left(F ;\left[c_{i}, d_{i}\right]\right)$ denotes the oscillation of $f$ over $\left[c_{i}, d_{i}\right]$; that is,

$$
\omega\left(F ;\left[c_{i}, d_{i}\right]\right)=\sup \left\{|F(x)-F(y)|: x, y \in\left[c_{i}, d_{i}\right]\right\} .
$$

A function $F$ is generalized absolutely continuous (or $\mathrm{ACG}^{*}$ ) on $E$ if $F$ is continuous on $E$ and if $E$ can be expressed as a countable union of sets on each of which $F$ is absolutely continuous (or $\mathrm{AC}^{*}$ ).

For the Lebesgue integral of function $f$, we denote that $\int_{0}^{t} f(s) d s$.

Denote by $C$ the continuous functions space on $[0,1]$, by AC the absolutely continuous functions space on $[0,1]$, by $\mathrm{ACG}^{*}$ the generalized absolutely continuous functions space on $[0,1]$, and by $H$ the space of HK-integrable functions from $[0,1]$ to $R$. Assume that the space $C$ is equipped with pointwise ordering and normed by the maximum norm, and that the space $H$ is equipped with a.e. pointwise ordering and normed by the Alexiewicz norm. 
The following Lemma 2.3-Lemma 2.7 are from [13, 14].

Lemma 2.3. The Henstock-Kurzweil integral is linear, and additive over nonoverlapping intervals of $[0,1]$.

Lemma 2.4. Let $f:[0,1] \rightarrow R$ be HK-integrable and let $g:[0,1] \rightarrow R$ be bounded variation. Then $f g$ is $H K$-integrable, and for every $t \in[0,1]$

$$
(H K) \int_{0}^{t} f(s) g(s) d s=g(t)(H K) \int_{0}^{t} f(s) d s-\int_{0}^{t}\left(g^{\prime}(s)(H K) \int_{0}^{s} f(\tau) d \tau\right) d s .
$$

Lemma 2.5. Let $f_{ \pm}:[0,1] \rightarrow R$ be HK-integrable. If $f_{-}(s) \leq f_{+}(s)$ for almost every $s \in[0,1]$, and if $[a, b] \subseteq[0,1]$, then

$$
(H K) \int_{a}^{b} f_{-}(s) d s \leq(H K) \int_{a}^{b} f_{+}(s) d s .
$$

Lemma 2.6. Let $f:[0,1] \rightarrow R$ be HK-integrable. Then the relation

$$
\tilde{f}(t)=c+(H K) \int_{0}^{t} f(s) d s, \quad t \in[0,1]
$$

defined a function $\tilde{f}:[0,1] \rightarrow R$, which is continuous and belongs to $A C G^{*}$, a.e. derivable and $\tilde{f}^{\prime}(t)=\underset{\widetilde{f}}{f}(t)$ a.e. on $[0,1]$.

$\tilde{f}$ is called a primitive of $f$.

Lemma 2.7. Assume that functions $f_{n}:[0,1] \rightarrow R, n \in N$ and $f_{ \pm}:[0,1] \rightarrow R$ are HK-integrable, that the sequence $\left\{f_{n}(s)\right\}$ is increasing (respectively decreasing) for almost every $s \in[0,1]$, and that

$$
f_{-}(s) \leq f_{n}(s) \leq f_{+}(s)
$$

for all $n \in N$ and a.e. $s \in[0,1]$. Then there exists such an HK-integrable function $f:[0,1] \rightarrow R$, that $f(s)=\lim _{n \rightarrow \infty} f_{n}(s)$ for a.e. $s \in[0,1]$, and that

$$
\lim _{n \rightarrow \infty}(H K) \int_{0}^{1} f_{n}(s) d s=(H K) \int_{0}^{1} f(s) d s .
$$

\section{Linear Problem}

We know that the homogeneous problem

$$
\begin{gathered}
-x^{\prime \prime}=0, \quad t \in(0,1), \\
x(0)-k_{1} x^{\prime}(0)=0, \\
x(1)+k_{2} x^{\prime}(1)=0,
\end{gathered}
$$


has only the trivial solution and Green's function is

$$
G(t, s)=\frac{1}{k_{1}+k_{2}+1} \begin{cases}\left(k_{1}+t\right)\left(k_{2}+1-s\right), & 0 \leq t \leq s \leq 1 \\ \left(k_{1}+s\right)\left(k_{2}+1-t\right), & 0 \leq s \leq t \leq 1\end{cases}
$$

It is easy to prove the following lemma.

Lemma 3.1. For every $t \in[0,1]$, functions $s \mapsto G(t, s)$ and $s \mapsto(\partial G / \partial t)(t, s)$ are derivable on $[0, t)$ and $(t, 1]$ and their derivations are absolutely continuous.

Lemma 3.2. Let $\sigma:[0,1] \rightarrow R$ be an HK-integrable function, then

(1) for every $t \in[0,1], G(t, s) \sigma(s)$ and $(\partial G / \partial t)(t, s) \sigma(s)$ are HK-integrable in s;

(2) the function, where $u_{\sigma}:[0,1] \rightarrow R$,

$$
u_{\sigma}(t)=(H K) \int_{0}^{1} G(t, s) \sigma(s) d s, \quad \forall t \in[0,1]
$$

is derivable a.e. on $[0,1]$ and

$$
u_{\sigma}^{\prime}(t)=(H K) \int_{0}^{1} \frac{\partial G}{\partial t}(t, s) \sigma(s) d s
$$

(3) $u_{\sigma}(t)$ satisfies the following conditions:

$$
\begin{aligned}
& u_{\sigma}(0)-k_{1} u_{\sigma}^{\prime}(0)=0, \\
& u_{\sigma}(1)+k_{2} u_{\sigma}^{\prime}(1)=0,
\end{aligned}
$$

(4) $u_{\sigma}^{\prime}(t)$ is derivable a.e. on $[0,1]$ and

$$
-u_{\sigma}^{\prime \prime}(t)=\sigma(t) \text {, a.e. in }[0,1]
$$

Proof. (1) From Lemma 3.1, since we know that $G(t, s)$ and $(\partial G / \partial t)(t, s)$ are absolutely continuous respect to $s$, and $\sigma(s) \in H$, the conclusions are in as follows. 
(2) Since

$$
\begin{aligned}
u_{\sigma}(t)= & (\mathrm{HK}) \int_{0}^{1} G(t, s) \sigma(s) d s \\
= & \frac{k_{1}+t}{k_{1}+k_{2}+1}(\mathrm{HK}) \int_{t}^{1}\left(k_{2}+1-s\right) \sigma(s) d s \\
& +\frac{k_{2}+1-t}{k_{1}+k_{2}+1}(\mathrm{HK}) \int_{0}^{t}\left(k_{1}+s\right) \sigma(s) d s,
\end{aligned}
$$

it follows from Lemma 2.6 that, for a.e. $t \in[0,1]$,

$$
\begin{aligned}
u_{\sigma}^{\prime}(t)= & \frac{1}{k_{1}+k_{2}+1}(\mathrm{HK}) \int_{t}^{1}\left(k_{2}+1-s\right) \sigma(s) d s-\frac{k_{1}+t}{k_{1}+k_{2}+1}\left(k_{2}+1-t\right) \sigma(t) \\
& -\frac{1}{k_{1}+k_{2}+1}(\mathrm{HK}) \int_{0}^{t}\left(k_{1}+s\right) \sigma(s) d s+\frac{k_{2}+1-t}{k_{1}+k_{2}+1}\left(k_{1}+t\right) \sigma(t) \\
= & (\mathrm{HK}) \int_{0}^{1} \frac{\partial G}{\partial t}(t, s) \sigma(s) d s .
\end{aligned}
$$

(3) Since

$$
\begin{aligned}
u_{\sigma}(0)= & \frac{k_{1}}{k_{1}+k_{2}+1}(\mathrm{HK}) \int_{0}^{1}\left(k_{2}+1-s\right) \sigma(s) d s, \\
u_{\sigma}^{\prime}(0)=\lim _{t \rightarrow 0^{+}} \frac{u_{\sigma}(t)-u_{\sigma}(0)}{t} & \\
= & \lim _{t \rightarrow 0^{+}} \frac{1}{t}\left[\frac{t}{k_{1}+k_{2}+1}(\mathrm{HK}) \int_{t}^{1}\left(k_{2}+1-s\right) \sigma(s) d s\right. \\
& \quad+\frac{k_{2}+1-t}{k_{1}+k_{2}+1}(\mathrm{HK}) \int_{0}^{t}\left(k_{1}+s\right) \sigma(s) d s \\
& \left.\quad-\frac{k_{1}}{k_{1}+k_{2}+1}(\mathrm{HK}) \int_{0}^{1}\left(k_{2}+1-s\right) \sigma(s) d s\right] \\
= & \frac{1}{k_{1}+k_{2}+1}(\mathrm{HK}) \int_{0}^{1}\left(k_{2}+1-s\right) \sigma(s) d s-\lim _{t \rightarrow 0^{+}} \frac{1}{t}(\mathrm{HK}) \int_{0}^{t} s \sigma(s) d s,
\end{aligned}
$$

we claim that

$$
\lim _{t \rightarrow 0^{+}} \frac{1}{t}(\mathrm{HK}) \int_{0}^{t} s \sigma(s) d s=0
$$


In fact, by Lemma 2.4,

$$
(\mathrm{HK}) \int_{0}^{t} s \sigma(s) d s=t(\mathrm{HK}) \int_{0}^{t} \sigma(s) d s-\int_{0}^{t}\left((\mathrm{HK}) \int_{0}^{s} \sigma(\tau) d \tau\right) d s
$$

Denote that $g(s)=(\mathrm{HK}) \int_{0}^{s} \sigma(\tau) d \tau$; then $g(s) \in \mathrm{ACG}^{*}$ and $\lim _{s \rightarrow 0} g(s)=0$. There exists $t_{0} \in[0, t]$ such that

$$
\int_{0}^{t} g(s) d s=g\left(t_{0}\right) t
$$

Therefore,

$$
\lim _{t \rightarrow 0^{+}} \frac{1}{t}(\mathrm{HK}) \int_{0}^{t} s \sigma(s) d s=\lim _{t \rightarrow 0^{+}}(\mathrm{HK}) \int_{0}^{t} \sigma(s) d s-\lim _{t \rightarrow 0^{+}} g\left(t_{0}\right)=0
$$

Thus, we have

$$
\begin{gathered}
u_{\sigma}^{\prime}(0)=\frac{1}{k_{1}+k_{2}+1}(\mathrm{HK}) \int_{0}^{1}\left(k_{2}+1-s\right) \sigma(s) d s, \\
u_{\sigma}(0)-k_{1} u_{\sigma}^{\prime}(0)=0 .
\end{gathered}
$$

The proof of another condition $u_{\sigma}(1)+k_{2} u_{\sigma}^{\prime}(1)=0$ is similar.

(4) Since

$$
u_{\sigma}^{\prime}(t)=\frac{1}{k_{1}+k_{2}+1}\left(-(\mathrm{HK}) \int_{0}^{t}\left(k_{1}+s\right) \sigma(s) d s+(\mathrm{HK}) \int_{t}^{1}\left(k_{2}+1-s\right) \sigma(s) d s\right)
$$

for a.e. $t \in[0,1]$, there exists a subset $I$ of $[0,1]$ with $\mu([0,1] \backslash I)=0$ such that $u_{\sigma}^{\prime}(t) \in \mathrm{ACG}^{*}$ on $I$. Relying on Lemma 2.6, $u_{\sigma}^{\prime}(t)$ is derivable a.e. on $I$ and, therefore, a.e. on $[0,1]$, and

$$
\begin{aligned}
-u_{\sigma}^{\prime \prime}(t) & =\frac{1}{k_{1}+k_{2}+1}\left(\left(k_{1}+t\right) \sigma(t)+\left(k_{2}+1-t\right) \sigma(t)\right) \\
& =\sigma(t), \quad \text { a.e. in }[0,1] .
\end{aligned}
$$


Theorem 3.3. Given functions $\sigma(t), \rho_{1}(t), \rho_{2}(t) \in H$. Then the following nonhomogeneous linear problem

$$
\begin{gathered}
-x^{\prime \prime}(t)=\sigma(t), \quad t \in(0,1), \\
x(0)-k_{1} x^{\prime}(0)=(H K) \int_{0}^{1} \rho_{1}(s) d s, \\
x(1)+k_{2} x^{\prime}(1)=(H K) \int_{0}^{1} \rho_{2}(s) d s,
\end{gathered}
$$

has a unique solution $x \in A C G^{*}$ and

$$
x(t)=p(t)+(H K) \int_{0}^{1} G(t, s) \sigma(s) d s,
$$

where

$$
p(t)=\frac{k_{2}+1-t}{k_{1}+k_{2}+1}(H K) \int_{0}^{1} \rho_{1}(s) d s+\frac{k_{1}+t}{k_{1}+k_{2}+1}(H K) \int_{0}^{1} \rho_{2}(s) d s
$$

Proof. We notice that $p(t) \in C^{2}[0,1]$ and

$$
\begin{gathered}
-p^{\prime \prime}(t)=0, \quad t \in(0,1), \\
p(0)-k_{1} p^{\prime}(0)=(\mathrm{HK}) \int_{0}^{1} \rho_{1}(s) d s, \\
p(1)+k_{2} p^{\prime}(1)=(\mathrm{HK}) \int_{0}^{1} \rho_{2}(s) d s .
\end{gathered}
$$

The facts associated with Lemma 3.2 deduce that the function $x(t)$ satisfies $x(t) \in \mathrm{ACG}^{*}, x^{\prime}(t)$ is derivable a.e. on $[0,1]$, and

$$
-x^{\prime \prime}(t)=\sigma(t), \quad \text { a.e. }[0,1]
$$

and $x(t)$ verifies the boundary conditions. The uniqueness of solution of (3.17) follows from Lemma 3.1. 
Journal of Inequalities and Applications

\section{The Nonlinear Problems}

In this section we consider the following nonlinear problems:

$$
\begin{gathered}
-x^{\prime \prime}=f(t, x), \quad t \in(0,1), \\
x(0)-k_{1} x^{\prime}(0)=\int_{0}^{1} h_{1}(s, x(s)) d s, \\
x(1)+k_{2} x^{\prime}(1)=\int_{0}^{1} h_{2}(s, x(s)) d s .
\end{gathered}
$$

We impose the following hypotheses on the functions $f$ and $h_{1}, h_{2}$.

$\left(H_{1}\right) f(t, x(t))$ and $h_{i}(t, x(t))(i=1,2)$ are HK-integrable whenever $x \in C$.

$\left(H_{2}\right) f(t, x)$ and $h_{i}(t, x)(i=1,2)$ are increasing in $x$ for almost every $t \in[0,1]$.

$\left(H_{3}\right)$ There exist HK-integrable functions $f^{ \pm}$and $h_{i}^{ \pm}(i=1,2)$ such that

$$
f^{-}(t) \leq f(t, x(t)) \leq f^{+}(t), \quad h_{i}^{-}(t) \leq h_{i}(t, x(t)) \leq h_{i}^{+}(t) \quad(i=1,2)
$$

a.e. hold on $[0,1]$ for all $x \in C$.

To prove our results, we need the following fixed point theorem for mappings of $C$ which is proved in [10].

Lemma 4.1. Let $G: C \rightarrow C$ be an increasing mapping which maps every monotone sequence $\left\{u_{n}\right\}$ of $C$ to a sequence $\left\{G u_{n}\right\}$ which converges pointwise to a function of $C$. If $u^{ \pm} \in C, u^{-} \leq u^{+}, u^{-} \leq G u^{-}$, and $\mathrm{Gu}^{+} \leq u^{+}$, then $G$ has in an order interval $\left[u_{-}, u^{+}\right]$of $C$ least and greatest fixed points and they are increasing in $G$.

We prove an existence result for solutions of (4.1).

Theorem 4.2. Assume that the hypotheses $\left(H_{1}\right)-\left(H_{3}\right)$ are satisfied, then (4.1) has least and greatest solutions in $A C G^{*}$.

Proof. We know from Theorem 3.3 that the solutions $x \in$ ACG $^{*}$ of (4.1) are the solutions of following operator equation:

$$
x(t)=(T x)(t)=(B x)(t)+(A x)(t), \quad x \in C,
$$

where

$$
\begin{aligned}
(B x)(t)= & \frac{k_{2}+1-t}{k_{1}+k_{2}+1}(\mathrm{HK}) \int_{0}^{1} h_{1}(s, x(s)) d s \\
& +\frac{k_{1}+t}{k_{1}+k_{2}+1}(\mathrm{HK}) \int_{0}^{1} h_{2}(s, x(s)) d s, \quad t \in[0,1] \\
(A x)(t)= & (\mathrm{HK}) \int_{0}^{1} G(t, s) f(s, x(s)) d s, \quad t \in[0,1] .
\end{aligned}
$$


The hypothesis $\left(H_{2}\right)$ and Lemma 2.5 imply that if $u, v \in C$ and $u \leq v$, then

$$
T u=B u+A u \leq B v+A v=T v .
$$

That is, $T$ is increasing in $C$.

Let $\left\{u_{n}\right\}$ be an increasing sequence in $C$, then the hypothess $\left(H_{1}\right)-\left(H_{3}\right)$ imply that the functions sequences $\left\{f\left(t, u_{n}(t)\right)\right\},\left\{h_{i}\left(t, u_{n}(t)\right)\right\}(i=1,2)$ are increasing in $n$ and belong to $H$, and

$$
\begin{gathered}
f^{-}(t) \leq f\left(t, u_{n}(t)\right) \leq f^{+}(t), \quad \text { a.e. } t \in[0,1], n \in N, \\
h_{i}^{-}(t) \leq h_{i}\left(t, u_{n}(t)\right) \leq h_{i}^{+}(t), \quad(i=1,2), \text { a.e. } t \in[0,1], n \in N, \\
(\mathrm{HK}) \int_{0}^{1} f^{-}(s) d s \leq(\mathrm{HK}) \int_{0}^{1} f\left(s, u_{n}(s)\right) d s \leq(\mathrm{HK}) \int_{0}^{1} f^{+}(s) d s, \quad n \in N, \\
(\mathrm{HK}) \int_{0}^{1} h_{i}^{-}(s) d s \leq(\mathrm{HK}) \int_{0}^{1} h_{i}\left(s, u_{n}(s)\right) d s \leq(\mathrm{HK}) \int_{0}^{1} h_{i}^{+}(s) d s, \quad(i=1,2), n \in N .
\end{gathered}
$$

Thus, by Lemma 2.7, there exist HK-integrable functions $v, w_{i}(i=1,2)$ such that

$$
\begin{gathered}
f\left(t, u_{n}(t)\right) \leq v(t), \quad f\left(t, u_{n}(t)\right) \longrightarrow v(t), \quad \text { a.e. } t \in[0,1], \\
h_{i}\left(t, u_{n}(t)\right) \leq w_{i}(t), \quad h_{i}\left(t, u_{n}(t)\right) \longrightarrow w_{i}(t), \quad(i=1,2), \text { a.e. } t \in[0,1], \\
\quad(\mathrm{HK}) \int_{0}^{1} f\left(s, u_{n}(s)\right) d s \longrightarrow(\mathrm{HK}) \int_{0}^{1} v(s) d s, \\
(\mathrm{HK}) \int_{0}^{1} h_{i}\left(s, u_{n}(s)\right) d s \longrightarrow(\mathrm{HK}) \int_{0}^{1} w_{i}(s) d s, \quad(i=1,2) .
\end{gathered}
$$

Denote that

$$
\begin{gathered}
q_{n}(t)=(\mathrm{HK}) \int_{0}^{1} G(t, s) f\left(s, u_{n}(s)\right) d s, \quad t \in[0,1], \\
q(t)=(\mathrm{HK}) \int_{0}^{1} G(t, s) v(s) d s, \quad t \in[0,1], \\
r_{n}(t)=\frac{k_{2}+1-t}{k_{1}+k_{2}+1}(\mathrm{HK}) \int_{0}^{1} h_{1}\left(s, u_{n}(s)\right) d s+\frac{k_{1}+t}{k_{1}+k_{2}+1}(\mathrm{HK}) \int_{0}^{1} h_{2}\left(s, u_{n}(s)\right) d s, \\
r(t)=\frac{k_{2}+1-t}{k_{1}+k_{2}+1}(\mathrm{HK}) \int_{0}^{1} w_{1}(s) d s+\frac{k_{1}+t}{k_{1}+k_{2}+1}(\mathrm{HK}) \int_{0}^{1} w_{2}(s) d s .
\end{gathered}
$$


Then we can easily get that $r_{n}(t) \rightarrow r(t)$ for every $t \in[0,1]$ and

$$
\begin{aligned}
0 \leq q(t)-q_{n}(t) & =(\mathrm{HK}) \int_{0}^{1} G(t, s)\left(v(s)-f\left(s, u_{n}(s)\right)\right) d s \\
& \leq G_{0}(\mathrm{HK}) \int_{0}^{1}\left(v(s)-f\left(s, u_{n}(s)\right)\right) d s \longrightarrow 0, \quad \forall t \in[0,1],
\end{aligned}
$$

which implies also that $q_{n}(t) \rightarrow q(t)$ for every $t \in[0,1]$. Therefore we obtain

$$
\left(T u_{n}\right)(t) \longrightarrow r(t)+q(t) \in C, \quad \forall t \in[0,1]
$$

Denoting that

$$
\begin{aligned}
x^{ \pm}(t)= & \frac{k_{2}+1-t}{k_{1}+k_{2}+1}(\mathrm{HK}) \int_{0}^{1} h_{1}^{ \pm}(s) d s+\frac{k_{1}+t}{k_{1}+k_{2}+1}(\mathrm{HK}) \int_{0}^{1} h_{2}^{ \pm}(s) d s \\
& +(\mathrm{HK}) \int_{0}^{1} G(t, s) f^{ \pm}(s) d s,
\end{aligned}
$$

then, by Lemma 2.6, $x^{ \pm} \in \mathrm{ACG}^{*}$. In addition, the hypothesis $\left(\mathrm{H}_{3}\right)$ implies that

$$
\begin{aligned}
& x^{-} \leq x^{+}, \\
& x^{-} \leq T x^{-}, \quad T x^{+} \leq x^{+} .
\end{aligned}
$$

Thus, by Lemma 4.1. We know that $T$ has in the order interval $\left[x^{-}, x^{+}\right]$of $C$ least fixed point $x_{*}$ and greatest fixed point $x^{*}$. The functions $x_{*}(t)$ and $x^{*}(t)$ are least and greatest solutions of (4.1) in $\left[x^{-}, x^{+}\right]$. The hypothesis $\left(H_{3}\right)$ implies also that if $x \in C$, then $T x \in\left[x^{-}, x^{+}\right]$. Thus all the solutions of (4.1) belong to the order interval $\left[x^{-}, x^{+}\right]$, whence $x_{*}(t)$ and $x^{*}(t)$ are least and greatest of all solutions in $C$ of (4.1).

On the other hand, if $x \in C$ is a solution of (4.1), then, from Lemma 2.6,

$$
\begin{aligned}
x(t)= & \frac{k_{2}+1-t}{k_{1}+k_{2}+1}(\mathrm{HK}) \int_{0}^{1} h_{1}(s, x(s)) d s+\frac{k_{1}+t}{k_{1}+k_{2}+1}(\mathrm{HK}) \int_{0}^{1} h_{2}(s, x(s)) d s \\
& +(\mathrm{HK}) \int_{0}^{1} G(t, s) f(s, x(s)) d s \in \mathrm{ACG}^{*} .
\end{aligned}
$$

The proof is completed. 


\section{An Example}

Consider the following problem:

$$
\begin{gathered}
-x^{\prime \prime}=g(t, x)+\sigma(t), \quad t \in(0,1), \\
x(0)=0 \\
x(1)=0
\end{gathered}
$$

where

$$
\sigma(t)=\frac{t^{2}}{(1-t)^{2}} \sin \frac{1}{t} \sin \frac{1}{1-t}-\frac{(1-t)^{2}}{t^{2}} \sin \frac{1}{t} \sin \frac{1}{1-t^{\prime}}
$$

and $g(t, x)$ satisfies the following caratheodory conditions:

$\left(L_{1}\right)$ the map $x \mapsto g(t, x)$ is continuous for a.e. $t \in[0,1]$,

$\left(L_{2}\right)$ the map $t \mapsto g(t, x)$ is measurable for all $x \in R$,

$\left(L_{3}\right)$ there exists $h \in L^{1}[0,1]$ with $\int_{0}^{1} h(t) d t<+\infty$ such that $|f(t, x)| \leq h(t)$ for a.e. $t \in$ $[0,1]$ and $x \in R$,

$\left(L_{4}\right) g(t, x)$ is increasing in $x$ for a.e. $t \in[0,1]$.

Since function $\sigma(t)$ is not Lebesgue integrable, the results in literature do not hold for (5.1). Let $f(t, x)=g(t, x)+\sigma(t), f^{ \pm}(t)= \pm h(t)+\sigma(t)$, then $f^{-}(t) \leq f(t, x) \leq f^{+}(t)$ and $f(t, x(t))$ is HK-integrable for every continuous $x$ since $g(t, x(t))$ is Lebesgue integrable for every continuous $x$ and $\sigma(t)$ HK-integrable.

Hence, the existence of continuous solution of problem (5.1) is guaranteed by Theorem 4.2.

\section{Acknowledgment}

This work was supported by NNSF of China (10871059).

\section{References}

[1] R. P. Agarwal and D. O'Regan, "Existence theory for single and multiple solutions to singular positone boundary value problems," Journal of Differential Equations, vol. 175, no. 2, pp. 393-414, 2001.

[2] D. Jiang, "Upper and lower solutions for a superlinear singular boundary value problem," Computers $\mathcal{E}$ Mathematics with Applications, vol. 41, no. 5-6, pp. 563-569, 2001.

[3] R. A. Khan, "The generalized method of quasilinearization and nonlinear boundary value problems with integral boundary conditions," Electronic Journal of Qualitative Theory of Differential Equations, no. 10, pp. 1-9, 2003.

[4] K. Lan and J. R. L. Webb, "Positive solutions of semilinear differential equations with singularities," Journal of Differential Equations, vol. 148, no. 2, pp. 407-421, 1998.

[5] A. Mao, S. Luan, and Y. Ding, "On the existence of positive solutions for a class of singular boundary value problems," Journal of Mathematical Analysis and Applications, vol. 298, no. 1, pp. 57-72, 2004. 
[6] S. Staněk, "Positive solutions of singular Dirichlet boundary value problems with time and space singularities," Nonlinear Analysis: Theory, Methods \& Applications, vol. 71, no. 10, pp. 4893-4905, 2009.

[7] S. D. Taliaferro, "A nonlinear singular boundary value problem," Nonlinear Analysis: Theory, Methods $\mathcal{E}$ Applications, vol. 3, no. 6, pp. 897-904, 1979.

[8] X. Zhang and L. Liu, "Positive solutions of superlinear semipositone singular Dirichlet boundary value problems," Journal of Mathematical Analysis and Applications, vol. 316, no. 2, pp. 525-537, 2006.

[9] S. Carl, S. Heikkilä, and G. Ye, "Order properties of spaces of non-absolutely integrable vector-valued functions and applications to differential equations," Differential and Integral Equations, vol. 22, no. 1-2, pp. 135-156, 2009.

[10] S. Heikkilä and M. Kumpulainen, "Monotone comvergence theorems for strongly Henstock-Kurzweil integrable operator-valued functions and applications," to appear.

[11] S. Heikkilä, M. Kumpulainen, and S. Seikkala, "Convergence theorems for HL integrable vectorvalued functions with applications," Nonlinear Analysis: Theory, Methods \& Applications, vol. 70, no. 5, pp. 1939-1955, 2009.

[12] B. Satco, "Second order three boundary value problem in Banach spaces via Henstock and HenstockKurzweil-Pettis integral," Journal of Mathematical Analysis and Applications, vol. 332, no. 2, pp. 919-933, 2007.

[13] S. Schwabik and G. Ye, Topics in Banach Space Integration, vol. 10 of Series in Real Analysis, World Scientific, Hackensack, NJ, USA, 2005.

[14] P. Y. Lee, Lanzhou Lectures on Henstock Integration, vol. 2 of Series in Real Analysis, World Scientific, Teaneck, NJ, USA, 1989. 